\title{
Prioritizing Road Network Connectivity Information for Disaster Response
}

\author{
Yingjie $\mathrm{Hu}$ \\ STKO Lab, Department of Geography \\ University of California Santa Barbara \\ yingjiehu@geog.ucsb.edu
}

\author{
Krzysztof Janowicz \\ STKO Lab, Department of Geography \\ University of California Santa Barbara \\ jano@geog.ucsb.edu
}

\begin{abstract}
Information plays an important role in disaster response. In the past, there has been a lack of up-to-date information following major disasters due to the limited means of communication. This situation has changed substantially in recent years. With the ubiquity of mobile devices, people experiencing emergency events may still be able to share information via social media and peer-to-peer networks. Meanwhile, volunteers throughout the world are remotely convened by humanitarian organizations to digitize satellite images for the impacted area. These processes produce rich information which presents a new challenge for decision makers who have to interpret large amount of heterogeneous information within limited time. This short paper discusses this problem and outlines a potential solution to prioritizing information in emergency situations. Specifically, we focus on information about road network connectivity, i.e., whether a road segment is still accessible after a disaster. We propose to integrate information value theory with graph theory, and prioritize information items based on their contributions to the successes of potential rescue tasks and to the more accurate estimation of road network connectivity. Finally, we point out directions for future work.
\end{abstract}

\section{Categories and Subject Descriptors}

H.1.1 [Systems and Information Theory]: [Value of information]; G.2.2 [Graph Theory]: [Network problems]

\section{Keywords}

Disaster Response, Information Value, Road Network, Graph Theory

\section{INTRODUCTION}

Information is a crucial resource in disaster response. After an emergency, decision makers rely on information to arrange rescue tasks and allocate relief resources. In the

Permission to make digital or hard copies of all or part of this work for personal or classroom use is granted without fee provided that copies are not made or distributed for profit or commercial advantage and that copies bear this notice and the full citation on the first page. To copy otherwise, to republish, to post on servers or to redistribute to lists, requires prior specific permission and/or a fee.

SIGSPATIAL'15, Nov 03-06, 2015, Seattle, Washington, USA .

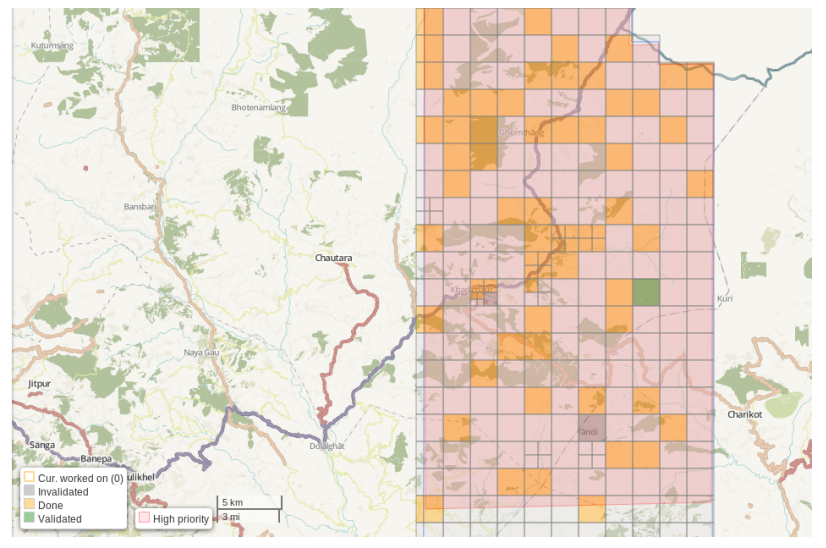

Figure 1: Collaborative mapping by volunteers after the Nepal earthquake in April 2015.

past, there has been a lack of up-to-date information following major disasters due to the limited means of communication. This situation has largely changed within the last few years. With the popularity of mobile devices, especially GPS-enabled devices, people experiencing disasters may still be able to share messages and locations on social media websites [9]. New applications also allow peer-to-peer communications in case of cell network failure. While such volunteered geographic information (VGI) has a varying quality, it can provide timely updates for estimating the disaster severity [4]. Meanwhile, volunteers around the globe can digitize remote sensing images of the impacted area. For example, after the Haiti earthquake in 2010, web portals were established for crisis mapping, and a large amount of digitized information was collected with the help of online volunteers $[12,8]$. A similar collaborative mapping was employed following the Nepal earthquake, in which the impacted area was divided into grids and crowdsourced to many volunteers (see Figure 1). With the availability of volunteercontributed information, many studies have been conducted to investigate this new data source for improving disaster response [10, 11].

The large amount of information, however, also presents a challenge to decision makers. After a disaster, information from different sources floods into the headquarters of response organizations. In such context, decision makers need to quickly interpret the information, estimate the different emergency levels, and decide which issues should be addressed immediately and which can be handled later. According to a report from the US National Public Radio 


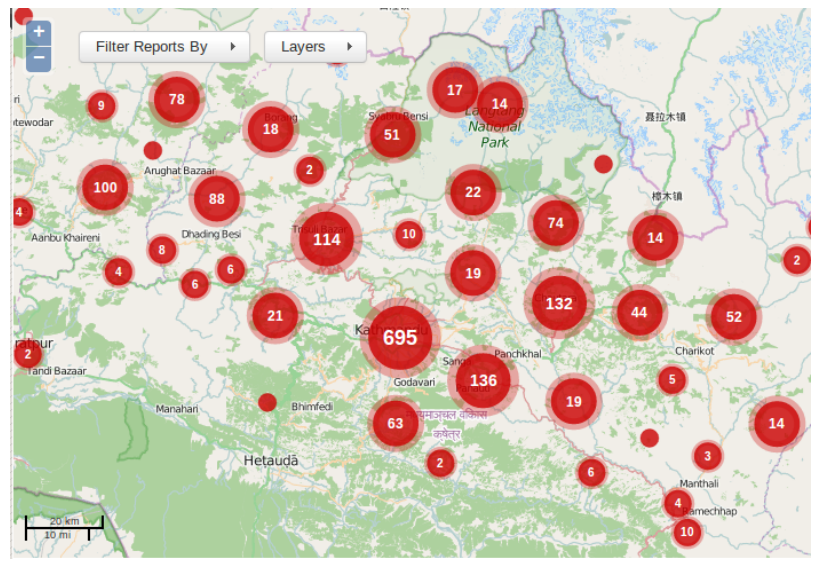

Figure 2: Information from different sources about the Nepal earthquake (http://quakemap.org).

during the response to the Nepal earthquake, thousands of volunteers were contributing information, and "aid groups sometimes get overwhelmed by the flood of digital data" . To facilitate processing, the contributed information is often aggregated and displayed on a map interface (see Figure 2 ). However, manual interpretation is still necessary to understand the content, which can be time-consuming when the volume of information is large and the update speed is high. In disaster response, timely rescue is critical for saving lives and reducing property loss. This leads to the question of whether one can design an algorithm to prioritize information items for disaster responders?

Answering the above question requires a method for measuring and quantifying the values of different information items in disaster response. The results can then be used to assign priorities to information items. Examining the role of information in disaster response is not new. For example, Comfort et al. (2004) employed agent-based model to simulate different disaster response scenarios, and concluded that information can contribute to increasing the efficiency of response activities [3]. Brooijmans (2008) took a qualitative approach to evaluate the added value of geographic information in disaster management, in which disaster responders from different sectors (e.g. firefighters, police, municipalities, and medics) were requested to answer questions through a questionnaire [1]. While these studies demonstrated the general usefulness of information in disaster response, they did not seek to differentiate the contributions from different information items.

In this short paper, we discuss the problem of information prioritization in the context of disaster response. Different types of information can be available in the disaster response process, and can contribute to decision making in different ways. In this work, we focus on road network connectivity information (i.e., whether a road segment is still accessible after a disaster), since this type of information can be commonly observed and is critical for planning rescue routes. More specifically, the research contribution of our work lies in the integration of information value theory with graph theory to prioritize different information items.

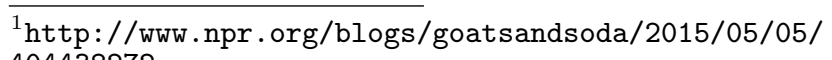
404438272
(1)

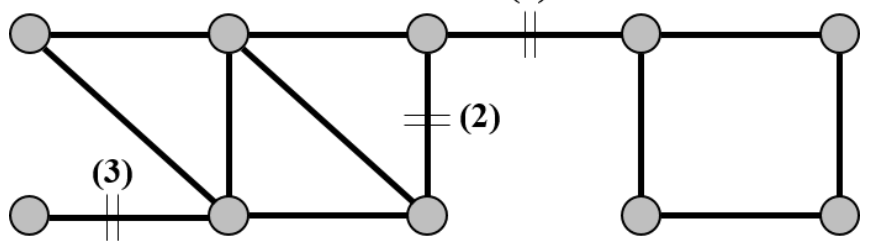

Figure 3: A road network of the impacted area.

In the following sections, we first briefly introduce information value theory and then outline the overall idea.

\section{INFORMATION VALUE THEORY}

Information value theory (IVT) was proposed by Ronald Howard back in 1966 [5]. In contrast to Shannon and Weaver's information theory that quantifies information by bits, IVT examines the value of information from a perspective of decision making. The value of information is then measured as the improvement introduced by the information into the decision making process. This improvement has been quantified using a utility function which assigns numeric values to the possible outcomes of a decision. Money, with its almost universal exchangeability, has been frequently used in utility functions, and as a result, the value of information has been often represented by a certain amount of money.

While IVT has been mathematically formalized in multiple ways in different studies, its core idea is to calculate the utility difference before and after an information item has been introduced into decision making. Such a broad notion can be represented by equation 1 .

$$
V(I)=U\left(d_{\text {after }}\right)-U\left(d_{\text {before }}\right)
$$

where $d_{\text {before }}$ represents the original decision before using information $I$, and $d_{\text {after }}$ represents the improved decision after $I$ has been used. $U$ is the utility function which quantifies the benefit of the decision, and $V(I)$ represents the information value of $I$. IVT has been widely applied to a variety of use cases, such as investment analysis [2] and clinical assessment [7]. In this work, we integrate IVT with graph theory, and measure the values of information in disaster response.

\section{PRELIMINARY METHOD}

The general idea of measuring the values of information on road network connectivity is to quantify the contribution of an information item to rescue tasks and the decision makers' estimation about the road network connectivity. Specifically, we use three simplified scenarios to illustrate our preliminary method in which an undirected graph $G(V, E)$ is used to represent the road network in the impacted area.

\subsection{Scenario 1}

Consider a scenario in Figure 3, in which three information items, $I_{1}, I_{2}$, and $I_{3}$, can inform the decision makers about the destruction of three road segments (1), (2), and (3) respectively. How can we assign priorities to the three information items?

We can transform this scenario into a bridge-finding problem. A bridge is an edge of a graph, whose removal will 
(1)

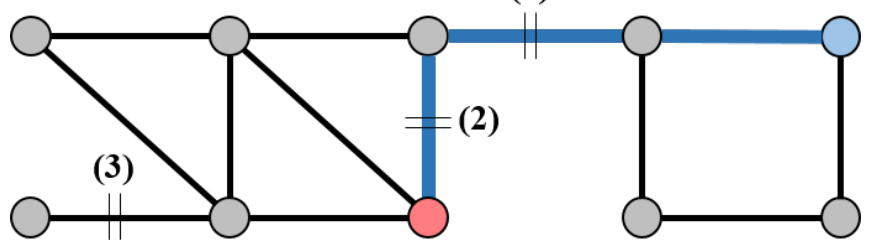

Figure 4: A road network of the impacted area with a rescue task.

increase the number of connected components of a graph. In Figure 3, segments (1) and (3) are bridges while (2) is not. Without up-to-date information, a decision maker may overestimate the connectivity of the road network, which could lead to ineffective response strategies. Thus, the value of information can be measured by its capability to reduce the overestimation of the decision maker. We define the maximum number of connected vertices among all subgraphs of $G$ as $v_{\max }$. Let $G_{1}, G_{2}, \ldots, G_{n}$ be the subgraphs of $G$, and $v_{1}, v_{2}, \ldots, v_{n}$ be the numbers of vertices in each of these subgraphs, $v_{\max }$ can be calculated as $\max _{i \in n} v_{i}$.

Let $v_{\max }^{b}$ be the maximum number of connected vertices the decision maker thinks the graph has before the information has been provided, and $v_{\max }^{a}$ be the number of vertices after the decision maker is aware of this information. The value of the information can then be measured as the difference between the two:

$$
V(I)=\left|v_{\max }^{a}-v_{\max }^{b}\right|
$$

To give concrete examples, $I_{2}$ is information about the connectivity of edge (2) (Figure 3 ), and removing this edge will not lead to a decrease of the maximum number of connected vertices. Thus, $V\left(I_{2}\right)=|10-10|=0$, indicating $I_{2}$ cannot directly contribute to reducing the decision maker's overestimation on the number of connected vertices (although $I_{2}$ does increase the number of vulnerable vertices; see scenario 3.). While both $I_{1}$ and $I_{3}$ provide information about bridges, segment (1) can largely reduce the maximum number of connected vertices in a subgraph, and thus $V\left(I_{1}\right)=|6-10|=4$. In contrast, $V\left(I_{3}\right)=|9-10|=1$. Therefore, the priorities of the three information items in this scenario should be: $I_{1}>I_{3}>I_{2}$.

\subsection{Scenario 2}

Consider another simplified scenario which adds a rescue task on top of the road connectivity case. Figure 4 illustrates this scenario. The blue vertex represents the location of a relief center and the red vertex is the site that needs help. Similarly, three information items, $I_{1}, I_{2}$, and $I_{3}$, are available, but how can we assign priorities to them?

First, we have to find the route that the rescue team is likely to take without any information, and such a route is represented as the blue line in Figure 4. Algorithms, such as Dijkstra or $A^{*}$, can be well used to find the shortest path. With the route found, we assign higher priorities to the information about the road segments on this route, since they can directly influence the success of the rescue task. In this scenario, we assign higher priorities to $I_{1}$ and $I_{2}$, although $I_{3}$ has a larger effect on reducing the decision makers' overestimation, as shown in scenario 1 . For $I_{1}$ and $I_{2}$, we can (a) (3) (b) (1)

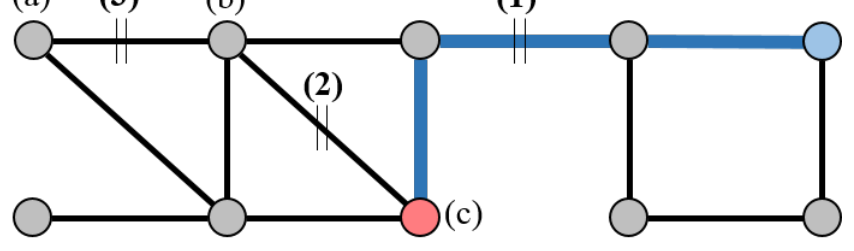

Figure 5: A road network of the impacted area with a rescue task and another two information items.

differentiate their IV based on their contributions to the rescue task. When no information is provided, the rescue team will be stopped at road segment (1), and there is no way for them to reach the target site. When only $I_{1}$ is provided, the rescue team will be informed about the critical disconnectivity at segment (1), and can request help for making segment (1) accessible (or partially accessible) before staring the rescue task. Although the rescue team will still be stopped at segment (2), they can change the route and will reach the target site. When only $I_{2}$ is provided, the rescue team will be informed about the disconnectivity at segment (2) but will not know about the critical disconnectivity at (1), and therefore will not be able to arrive at the target location. Given the road network, we can measure the length of the final route. We define the special situation in which the rescue team cannot be re-routed as having an infinite route length (i.e., $+\infty$ ). Thus, the value of information can be calculated as the difference of route length before and after information has been provided:

$$
V(I)=\left|l_{\text {best }}^{a}-l_{\text {best }}^{b}\right|
$$

where $l_{\text {best }}^{b}$ and $l_{\text {best }}^{a}$ are the actual lengths of the shortest path before and after this information is given. In practice, roads can be repaired or alternative means of transportation can be deployed. Thus, one can assign a substantial penalty (say 1000) instead of $+\infty$ and thereby, $V\left(I_{1}\right)=|4-1000|=$ 996 and $V\left(I_{2}\right)=|1000-1000|=0$. We have assumed that each road segment has a length of 1 , and different weights could also be used in more complex simulation. Hence, in this scenario, the priorities of information are: $I_{1}>I_{2}>I_{3}$.

\subsection{Scenario 3}

This scenario discusses a situation in which a rescue task is still provided but $I_{2}$ and $I_{3}$ are about the connectivities of two other road segments (Figure 5). From scenario 2, we can decide that $I_{1}$ has the highest priority, but how could we differentiate the relative values of $I_{2}$ and $I_{3}$ ?

It can be seen that neither segment (2) nor (3) are bridges in graph $G$, and also neither of them is on the rescue route. To determine the relative importances of the two information items, we propose to evaluate their capabilities in reducing a decision maker's overestimation on the network connectivity. In contrast to scenario 1 , we examine the numbers of vulnerable vertices estimated by the decision maker before and after an information item is provided. This road network vulnerability analysis has also been discussed in the transportation literature [6]. We define vulnerable vertices as those that connect to other vertices through only a single edge, and thus, can be easily disconnected from the graph. For example, in Figure 5, segment (3) connects vertices (a) 
and (b), and removing segment (3) will make (a) a vulnerable vertex. In contrast, when segment (2) is removed, neither (b) nor (c) will become vulnerable. Let $v_{v u l}^{b}$ and $v_{v u l}^{a}$ be the numbers of vulnerable vertices estimated by the decision maker before and after the information has been provided. The value of the information can be quantified as the difference between the two (equation 4). Since $I_{3}$ can help decision makers reach more accurate estimation about the vulnerability of the road network, the priorities of the three information items should be: $I_{1}>I_{3}>I_{2}$.

$$
V(I)=\left|v_{v u l}^{a}-v_{v u l}^{b}\right|
$$

\subsection{Combined Information Values}

Most situations will require a combined approach with multiple steps. First, one would identify the information about the road segments which are on the potential routes of the rescue tasks. Such information could be assigned higher priorities since they are important for the successes of rescue tasks, and the relative priorities among the information items in this group can be determined as in scenario 2. The second step identifies the information items which are about graph bridges in the road network. Such information is also important since they can largely reduce the decision makers' overestimation on the road network connectivity, and the relative values of these information items can be determined similarly as in scenario 1 . In the last step, we examine the remained information items, and quantify their values by counting the numbers of vertices that could be left vulnerable, as in scenario 3 .

\section{FUTURE DIRECTIONS}

In this short paper, we have outlined the problem of information prioritization in disaster response. We have focused on one type of information, namely road network connectivity information, and proposed to integrate information value theory with graph theory to quantify information values. Particularly, we have examined the contributions of information on the successes of rescue tasks and on reducing the decision maker's overestimation of the road network connectivity. The presented work is a first step and the following directions should be pursued in future:

- Representation of road networks. We have used an undirected graph to represent the road network, which is a simplified model that neither considers the vertex types nor the directions of the roads. In reality, these factors can influence the values of road connectivity information (e.g., the vertex may be a hospital, or the road is only disconnected in one direction). Thus, future research could employ a more complex representation to model the road network more accurately.

- Consequences of incorrect prioritization. Prioritizing information items carries the risk of making mistakes, which may have different consequences. It can be expected that false positives, i.e., less important information that has been assigned higher priorities, should be tolerable, whereas false negatives, i.e., more important information that has been given low priorities, may have more severe consequences. While it is not possible to avoid errors in the presence of false, low-quality, or missing information, we could develop suitable cost functions to reduce the chance of false negatives.

- Evaluation. While the introduced method can assign priorities to information items, it does not answer the question of how to verify the suitability of the prioritization in real emergencies. One possible approach is to conduct human participant experiments. Disaster responders, such as firefighters and policemen, could be invited to evaluate the prioritized information. However, such experiments can be time-consuming and may not scale up to many participants. Thus, it would be interesting to see whether it is possible to perform evaluation based on data recorded from previous disaster responses.

\section{REFERENCES}

[1] P. Brooijmans. How to measure the added value of geographic information in Disaster Management. $\mathrm{PhD}$ thesis, Free University, Amsterdam, 2008.

[2] C. J. Chen, S. Chen, and X. Su. Is accounting information value-relevant in the emerging chinese stock market? Journal of International Accounting, Auditing and Taxation, 10(1):1-22, 2001.

[3] L. K. Comfort, K. Ko, and A. Zagorecki. Coordination in rapidly evolving disaster response systems the role of information. American Behavioral Scientist, 48(3):295-313, 2004.

[4] M. F. Goodchild and J. A. Glennon. Crowdsourcing geographic information for disaster response: a research frontier. International Journal of Digital Earth, 3(3):231-241, 2010.

[5] R. A. Howard. Information value theory. IEEE Transactions on Systems Science and Cybernetics, 2(1):22-26, 1966

[6] E. Jenelius, T. Petersen, and L.-G. Mattsson. Importance and exposure in road network vulnerability analysis. Transportation Research Part A: Policy and Practice, 40(7):537-560, 2006.

[7] R. M. McFall and T. A. Treat. Quantifying the information value of clinical assessments with signal detection theory. Annual review of psychology, 50(1):215-241, 1999.

[8] P. Meier. New information technologies and their impact on the humanitarian sector. International review of the Red Cross, 93(884):1239-1263, 2011.

[9] S. E. Middleton, L. Middleton, and S. Modafferi. Real-time crisis mapping of natural disasters using social media. Intelligent Systems, IEEE, 29(2):9-17, 2014.

[10] K. Poser and D. Dransch. Volunteered geographic information for disaster management with application to rapid flood damage estimation. Geomatica, 64(1):89-98, 2010.

[11] A. H. Tapia, K. Bajpai, B. J. Jansen, J. Yen, and L. Giles. Seeking the trustworthy tweet: Can microblogged data fit the information needs of disaster response and humanitarian relief organizations. In 8 th Inter. ISCRAM Conference, pages 1-10, 2011.

[12] M. Zook, M. Graham, T. Shelton, and S. Gorman. Volunteered geographic information and crowdsourcing disaster relief: a case study of the haitian earthquake. Available at SSRN 2216649, 2010. 